\title{
Asymptotic Expansions of Feynman Amplitudes in a Generic Covariant Gauge
}

\author{
C.A. Linhares ${ }^{a}$, A.P.C. Malbouisson ${ }^{b}$ and I. Roditi ${ }^{b}$ \\ ${ }^{a}$ Instituto de Física, Universidade do Estado do Rio de Janeiro, \\ Rua São Francisco Xavier, 524, 20559-900 Rio de Janeiro, RJ, Brazil and \\ ${ }^{b}$ Centro Brasileiro de Pesquisas Físicas, \\ Rua Dr. Xavier Sigaud, 150, \\ 22290-180 Rio de Janeiro, RJ, Brazil
}

\begin{abstract}
We show in this paper how to construct Symanzik polynomials and the Schwinger parametric representation of Feynman amplitudes for gauge theories in a generic covariant gauge. The complete Mellin representation of such amplitudes is then established in terms of invariants (squared sums of external momenta and squared masses). From the scaling of the invariants by a parameter we extend for the present situation a theorem on asymptotic expansions, previously proven for the case of scalar field theories, valid for both ultraviolet or infrared behaviors of Feynman amplitudes.
\end{abstract}

\section{INTRODUCTION}

Asymptotic expansions for amplitudes related to Feynman diagrams continue to be a topic of interest. This is specially true in what regards the infrared and ultraviolet behaviors of gauge theories, studies of critical phenomena and attempts to understand confinement in the field-theoretical framework of quantum chromodynamics. With respect to gauge theories, whose interaction mediator field has a propagator of the form (in Euclidean space)

$$
\frac{1}{k^{2}+\mu^{2}}\left(\delta_{\mu \nu}-(1-\xi) \frac{k_{\mu} k_{\nu}}{k^{2}+\mu^{2}}\right)
$$

where $\mu$ is an infrared mass regulator and $\xi$ is the gauge-fixing parameter, amplitude computations are usually performed by choosing, for simplicity, the $(\xi=1)$ Feynman gauge. This has been done, for example, in a recent paper directly connected to the subject of our interest [1], where its authors were able to produce asymptotic expansions for some 2- and 3-loop vertex corrections in QED, using a method based on the Mellin-Barnes transform of Feynman amplitudes. There are, however, some circumstances in which it is desirable to work with other choices of $\xi$, normally involving non-gauge-invariant quantities. This is the case, for instance, of recent discussions [2, 3] on the form factors of gluon and ghost propagators in infrared QCD, whose computations cannot be formulated in the Feynman gauge. Also, in the context of noncommutative supersymmetric field theories, some calculations of radiative corrections require the choice of a non-Feynman gauge [4]. The intrinsic complexity of working in a general gauge, and its relevance for physical situations as those just mentioned, have led us to consider establishing asymptotic expressions in a general covariant regime. We shall do this by using the method of the complete Mellin representation of Feynman amplitudes. It shares with the Mellin transform method of Ref. [1] the advantage of avoiding the explicit calculation of amplitudes before deriving the asymptotic expressions.

Thus, in the present paper, we wish to extend the method when one considers the amplitudes in a generic covariant gauge. We here intend to take up the long tradition that started in the 1970's with the papers [5, 6, 7] within the Bogoliubov-Parasiuk-Hepp-Zimmermann formalism. Their approach is based on the Schwinger representation of amplitudes. This representation has the advantage of being expressed in terms of so-called Symanzik polynomials in the Schwinger parameters, which can be deduced in a straightforward way from the topological characteristics of the diagrams one is interested in. From the topological point of view, the diagrams are considered as a set of interconnected lines, ignoring distinctions between those related to gauge fields and those to matter fields. These 'topological formulas' are thus determined from inspection of the 1-trees and 2-trees constructed from the diagram by omitting the adequate number of lines [8, 9]. The Mellin transform technique was then used to prove theorems leading to the asymptotic expansion of convergent Feynman diagrams. External momenta are scaled by some parameter $\lambda$ and through the Mellin transform with respect to the scaling parameter, as one takes the limit of $\lambda$ going to infinity, the existence of an asymptotic series in powers of $\lambda$ and powers of logarithms of $\lambda$ is proved, and the series coefficients computed. Divergent diagrams were treated in [10].

In the subsequent years, the subject had further advancements, with the inclusion of the internal squared masses as possible invariants which may be scaled, in order to study the infrared behavior of the amplitudes as well [11]. There the concept of 'FINE' polynomials was introduced, that is, those having the property of being factorizable in each Hepp sector [12] of the variables. It was then argued that they may be 'desingularized', which means that the integrand has a meromorphic structure, so that the residues of its various poles may be obtained. However, this is not the general situation, and in many diagrams the FINE property does not occur, which is required to hold by the Mellin transform method. A first solution to this problem was presented in Ref. [11] by introducing the so-called 'multiple 
Mellin' representation, which consists essentially in splitting the Symanzik polynomials in a certain number of pieces, each one of which having the FINE property. Then, after scaling by the parameter $\lambda$, an asymptotic expansion can be obtained as a sum over all Hepp sectors. This is always possible to do, if one adopts, as was done in Refs. 13, 14, 15], the extreme point of view which consists in splitting the Symanzik polynomials in all its monomials. Moreover, this apparent complication is compensated by the fact that one can dispense with the use of Hepp sectors altogether. This came to be known as the 'complete Mellin' (CM) representation.

The CM representation provides a general proof of the existence of an asymptotic expansion in powers and powers of logarithms of the scaling parameter and no change of variables is needed: not only the Schwinger variables provide a desingularization, but the integrations over such variables can be explicitly performed, and we are left with the pure geometrical study of convex polyhedra in the Mellin variables. It has been proven [13] that the same results of [11] are obtained in a simpler way, and asymptotic expansions are computed in a more compact form, without any division of the integral into the Hepp sectors. Another feature of the CM representation is that it allows a unified treatment of the asymptotic behavior of both ultraviolet convergent and divergent amplitudes. Indeed, as is shown in Refs. [13, 14], the renormalization procedure does not alter the algebraic structure of integrands in the CM representation. It only changes the set of relevant integration domains in the Mellin variable space.

Other applications of the method included a study of the infrared behavior of amplitudes relevant to critical phenomena [16] and of dimensional regularization [15].

In Sec. II of the present paper, we show how to write down a Schwinger representation for a Feynman amplitude, when the gauge-fixing parameter $\xi$ is left unchosen, through the use of a 'topological formula'. In this case, then, as seen in eq. (11), every gauge propagator in a diagram contributes with a second term with momentum dependence $\left(k^{2}+\mu^{2}\right)^{-2}$, in addition to the $\left(k^{2}+\mu^{2}\right)^{-1}$ dependence of the first term, the latter one being the same that occurs in scalar theories and gauge theories in the Feynman gauge. This fact entails the introduction of two Schwinger parameters to the same gauge-field internal line of the diagram. Still, one should consider the momentum dependence occuring in the numerator, coming not only from the gauge-field propagator, but also from those of fermionic particles, and, in principle, from momentum-dependent vertices and counterterms. Numerators, however, modify the topological formulas in a systematic way [5, 7, 14].

Sec. III reviews the complete Mellin transform method, and we demonstrate in Sec. IV the existence of the asymptotic series for gauge theories in our generalized case. In Sec. V we present our conclusions.

The Euclidean metric will be used throughout the paper.

\section{PARAMETRIC REPRESENTATION AND TOPOLOGICAL FORMULAS}

We start by considering an arbitrary connected Feynman diagram $G$ in a purely scalar theory and let us denote by $I_{G}$ its contribution to some $n$-point Green's function in momentum space. It is a function of the relativistic invariants built from the external momenta and squared masses. Topologically, the Feynman diagram $G$ is a set of $I$ internal lines, $V$ vertices and $L$ loops and these quantities are well-known to satisfy the relation $L=I-V+1$. A $q$-tree of the diagram $G$ is a subdiagram of $G$ having $q$ connected components, without loops, and linking all the vertices of $G$. Of particular interest for us are the cases of $q=1$ (1-trees) and $q=2$ (2-trees).

Let us define $P_{v}$ as the sum of all external momenta that end at the vertex $v$ and let us assume that they are such that $\sum_{v=1}^{V} P_{v}=0$. Apart from global momentum conservation and overall constant factors coming from the vertices (for instance, such as $(-i \lambda)^{V}$ in $\lambda \phi^{4} / 4$ ! theory) or from a possible symmetry factor, the arbitrary amplitude reads

$$
I_{G}\left(P_{v} ; m_{l}^{2}\right)=\int \prod_{l=1}^{I} \frac{d^{d} k_{l}}{(2 \pi)^{d}} \frac{1}{k_{l}^{2}+m_{l}^{2}} \prod_{v=1}^{V}(2 \pi)^{d} \delta^{d}\left(P_{v}-\sum_{l=1}^{I} \epsilon_{v l} k_{l}\right) .
$$

In this formula, we integrate the Euclidean propagators (with possibly different masses $m_{l}$ ) over all momenta $k_{l}$ associated to each internal line $l$. The last factor ensures momentum conservation at each vertex. In it we have introduced the so-called incidence matrix, which is defined as

$$
\epsilon_{v l}= \begin{cases}+1 & \text { if the vertex } v \text { is the starting point of line } l \\ -1 & \text { if the vertex } v \text { is the ending point of line } l \\ 0 & \text { otherwise. }\end{cases}
$$

The Schwinger parametric integral $\alpha$-representation is introduced by expressing each propagator in the form

$$
\frac{1}{k^{2}+m^{2}}=\int_{0}^{\infty} d \alpha e^{-\alpha\left(k^{2}+m^{2}\right)}
$$


and the delta function is expressed as an integral as well,

$$
(2 \pi)^{d} \delta^{d}\left(P_{v}-\sum_{l=1}^{I} \epsilon_{v l} k_{l}\right)=\int d^{d} y e^{-i y \cdot\left(P_{v}-\sum_{l} \epsilon_{v l} k_{l}\right)} .
$$

Upon integration over each $k_{l}$ and then over the $y$ variables, the amplitude is finally expressed as [8, 9]

$$
I_{G}\left(P_{v} ; m_{l}^{2}\right)=\int_{0}^{\infty} \frac{\prod_{l=1}^{I} d \alpha_{l}}{(4 \pi)^{d L / 2} U^{d / 2}(\alpha)} e^{-\sum_{l} \alpha_{l} m_{l}^{2}} e^{-N\left(s_{K} ; \alpha\right) / U(\alpha)}
$$

where $U$ and $N$ are homogeneous polynomials in the $\alpha_{l}$ parameters, of order $L$ and $L+1$, respectively, constructed with topological relations defined by the 1- and 2-trees of the diagram $G$ :

$$
U(\alpha)=\sum_{T} \prod_{l \notin T} \alpha_{l}, \quad N(\alpha)=\sum_{K} s_{K}\left(\prod_{l \notin K} \alpha_{l}\right) .
$$

The symbols $\sum_{T}$ and $\sum_{K}$ denote, respectively, summation over the 1-trees $T$ and 2-trees $K$ of $G$. $s_{K}$ is the cut-invariant of one of the two connected pieces of the 2-tree $K$, that is, the square of the total external momentum $P_{v}$ entering one of pieces of $K$ (any one of them equivalently, by momentum conservation). $U$ and $N$ are known in the literature as the Symanzik polynomials.

Let us consider now a Yukawa-type scalar-fermion theory. A generic amplitude contains a certain number of fermion propagators which introduce a momentum-dependent polynomial $\mathcal{P}\left(k_{l}\right)=\prod_{l}\left(k_{l}-m_{f}\right)$ as a numerator in its expression, $m_{f}$ being the fermion mass. Differently from eq. (2), the amplitude now is expressed as

$$
I_{G}\left(P_{v} ; m_{l}^{2}\right)=\int\left(\prod_{l=1}^{I} \frac{d^{d} k_{l}}{(2 \pi)^{d}}\right) \frac{\mathcal{P}\left(k_{l}\right)}{k_{l}^{2}+m_{l}^{2}} \prod_{v=1}^{V}(2 \pi)^{d} \delta^{d}\left(P_{v}-\sum_{l=1}^{I} \epsilon_{v l} k_{l}\right) .
$$

The internal line index $l$ runs indistinctively over scalar or fermionic lines and $m_{l}$ is to be taken either as the scalar field mass or the fermion mass, accordingly. If we take the integral representation [5, 7, 14]

$$
\frac{\mathcal{P}\left(k_{l}\right)}{k_{l}^{2}+m_{l}^{2}}=\left.\int_{0}^{\infty} d \alpha_{l} \mathcal{P}\left(-\frac{1}{\alpha_{l}} \frac{\partial}{\partial \zeta_{l}}\right) e^{-\alpha_{l}\left(k_{l}^{2}+m_{l}^{2}+k_{l} \cdot \zeta_{l}\right)}\right|_{\zeta_{l}=0},
$$

the integrations in the amplitude may be performed in a similar way as in the purely scalar case, with the result that

$$
I_{G}\left(P_{v} ; m_{l}^{2}\right)=\left.\int_{0}^{\infty} \frac{\prod_{l=1}^{I} d \alpha_{l}}{(4 \pi)^{d L / 2} U^{d / 2}(\alpha)} \mathcal{P}\left(-\frac{1}{\alpha_{l}} \frac{\partial}{\partial \zeta_{l}}\right) e^{-\sum_{l} \alpha_{l} \bar{m}_{l}^{2}} e^{-N\left(\bar{s}_{K} ; \alpha\right) / U(\alpha)}\right|_{\zeta_{l}=0}
$$

where

$$
\bar{m}_{l}^{2}=m_{l}^{2}-\zeta_{l}^{2} / 4
$$

and $\bar{s}_{K}$ is the same cut-invariant of the 2 -tree $K$ that occurs in (7), for which the external momenta $P_{v}$ is replaced by

$$
\bar{P}_{v}=P_{v}+\sum_{l} \epsilon_{v l} \zeta_{l} / 2
$$

Let us now pass to our main subject, fermionic gauge theories. For a given generic $\operatorname{diagram} G$, let us call $F$ the set of its (fermionic) matter field lines and $\Gamma$ the set of its gauge-field propagators. The set of all lines in $G$ is denoted by $E=F \cup \Gamma$. The number of lines in each of these sets are denoted by $N_{F}$ and $N_{\Gamma}$, respectively, and therefore the total number of propagators is $N_{E}=N_{F}+N_{\Gamma}$. In an arbitrary gauge, using the gauge-field propagator (1), the amplitude $I_{G}$, related to the diagram $G$, is

$$
I_{G}(\xi)=C_{G} \int\left(\prod_{l=1}^{L} \frac{d^{d} k_{l}}{(2 \pi)^{d}}\right) \prod_{i=1}^{N_{F}} \mathcal{P}\left(q_{i}\right) \frac{1}{q_{i}^{2}+m_{i}^{2}} \prod_{j=1}^{N_{\Gamma}}\left(\frac{1}{p_{j}^{2}+\mu^{2}}-(1-\xi) \frac{p_{j} p_{j}}{\left(p_{j}^{2}+\mu^{2}\right)^{2}}\right),
$$

which, in the simpler case of the Feynman gauge, reduces to

$$
I_{G}(\xi=1)=C_{G} \int\left(\prod_{l=1}^{L} \frac{d^{d} k_{l}}{(2 \pi)^{d}}\right) \prod_{i=1}^{N_{F}} \mathcal{P}\left(q_{i}\right) \frac{1}{q_{i}^{2}+m_{i}^{2}} \prod_{j=1}^{N_{\Gamma}} \frac{1}{p_{j}^{2}+\mu^{2}} .
$$


To deal with the general expression, we recast it in the form

$$
I_{G}(\xi)=C_{G} \int\left(\prod_{l=1}^{L} \frac{d^{d} k_{l}}{(2 \pi)^{d}}\right) \prod_{i=1}^{N_{F}} \mathcal{P}\left(q_{i}\right) \frac{1}{q_{i}^{2}+m_{i}^{2}} \prod_{j=1}^{N_{\Gamma}} \mathcal{Q}\left(\xi, p_{j}\right) \frac{1}{\left(p_{j}^{2}+\mu^{2}\right)^{2}},
$$

where $\mathcal{Q}$ is defined as $\prod_{j=1}^{N_{\Gamma}}\left(p_{j}^{2}+\mu^{2}-(1-\xi) p_{j} p_{j}\right)$, thus carrying the dependence on the gauge choice. We see that $I_{G}(\xi=1)$ has a similar structure with respect to that of the Yukawa case above. Here, we collect in $C_{G}$ all global factors; the parameters $m_{i}$ are the (possibly different) matter-field masses and $\mu$ is the gauge-field infrared regulator. As before, let $P$ denote the set of external momenta; thus, to shorten the expression, we have considered the momenta $q_{i}\left(P,\left\{k_{l}\right\}\right)$ and $p_{j}\left(P,\left\{k_{l}\right\}\right)$ as linear functions of the set of $k_{l}$ obtained by momentum conservation at each vertex. $\mathcal{P}$ and $\mathcal{Q}$ are polynomials in the momenta, coming from the numerators of the propagators. They also contain factors such as the metric tensor and Dirac matrices, appropriate for a given diagram $G$ (spacetime indices are omitted).

For both of the integrals above, a Schwinger parametric representation can be written down. In order to do this, we particularize eq. (9) for each of the numerators in the integrals (14) and (15). Integral (14) has an analogous representation like in Yukawa theory, eq. (6), in which a vector $\zeta_{i}$ is attached to each fermionic line $i$ such that the momenta in the $\mathcal{P}$ polynomial are substituted by a corresponding derivative with respect to $\zeta_{i}$ at $\zeta_{i}$ equal to zero. We can then write the Feynman-gauge amplitude as

$$
I_{G}(\xi=1)=\left.C_{G} \int_{0}^{\infty} \frac{\prod_{l=1}^{N_{E}} d \alpha_{l}}{(4 \pi)^{d L / 2} U^{d / 2}} \prod_{i \in F} \mathcal{P}\left(-\frac{1}{\alpha_{i}} \frac{\partial}{\partial \zeta_{i}}\right) e^{-\sum_{i=1}^{N_{F}} \bar{m}_{i}^{2} \alpha_{i}-\mu^{2} \sum_{j=1}^{N_{\Gamma}} \alpha_{j}} e^{-N\left(\bar{s}_{K}\right) / U}\right|_{\zeta_{l}=0},
$$

with the Symanzik polynomials given by the same definitions as above, eqs. (7), modified by the presence of the auxiliary variables $\zeta_{l}$, just as in the scalar-fermion case, eqs. (11) and (12).

For the general case, we remind that the starting point for the parametric representation is eq. (44). Therefore, in eq. (15), as we have products of $\left(p_{j}+\mu^{2}\right)^{2}$ in the denominator, we should associate two Schwinger parameters, say, $\beta_{1}$ and $\beta_{2}$, to the same line, that is,

$$
\frac{1}{\left(p^{2}+\mu^{2}\right)^{2}}=\left(\int_{0}^{\infty} d \beta_{1} e^{-\beta_{1}\left(p^{2}+\mu^{2}\right)}\right)\left(\int_{0}^{\infty} d \beta_{2} e^{-\beta_{2}\left(p^{2}+\mu^{2}\right)}\right) .
$$

This means that the set of all lines of the diagram, related to the momenta $q_{i}$ and $p_{j}$, is such that to each matter-field line $i$ is attributed a parameter $\alpha_{i}$, whereas for each gauge-field line $r$ is associated a couple of parameters $\beta_{r_{1}}, \beta_{r_{2}}$. Thus the parametric representation for $I_{G}(\xi)$ has the form

$$
\begin{aligned}
I_{G}(\xi)= & C_{G} \int_{0}^{\infty} \frac{\prod_{i=1}^{N_{F}} d \alpha_{i} \prod_{r_{1}, r_{2}=1}^{N_{\Gamma}} d \beta_{r_{1}} d \beta_{r_{2}}}{(4 \pi)^{d L / 2} U^{d / 2}} \\
& \times \prod_{i \in F} \mathcal{P}\left(-\frac{1}{\alpha_{i}} \frac{\partial}{\partial \zeta_{i}}\right) \prod_{r_{1} \in \Gamma} \mathcal{Q}_{1}\left(-\frac{1}{\beta_{r_{1}}} \frac{\partial}{\partial \zeta_{r_{1}}}\right) \prod_{r_{2} \in \Gamma} \mathcal{Q}_{2}\left(-\frac{1}{\beta_{r_{2}}} \frac{\partial}{\partial \zeta_{r_{2}}}\right) \\
& \times\left. e^{-\sum_{i=1}^{N_{F}} \bar{m}_{i}^{2} \alpha_{i}-\sum_{r_{1}=1}^{N_{\Gamma}} \bar{\mu}_{r_{1}}^{2} \beta_{r_{1}}-\sum_{r_{2}=1}^{N_{\Gamma}} \bar{\mu}_{r_{2}}^{2} \beta_{r_{2}}} e^{-N\left(\bar{s}_{K}\right) / U}\right|_{\zeta=0} .
\end{aligned}
$$

where now

$$
U=\sum_{T} \prod_{i \notin T} \alpha_{i} \prod_{r_{1} \notin T} \beta_{r_{1}} \prod_{r_{2} \notin T} \beta_{r_{2}}, \quad N\left(\bar{s}_{K}\right)=\sum_{K} \bar{s}_{K} \prod_{i \notin K} \alpha_{i} \prod_{r_{2} \notin T} \beta_{r_{1}} \prod_{r_{2} \notin T} \beta_{r_{2}} .
$$

\section{THE COMPLETE MELLIN REPRESENTATION FOR SCALAR THEORIES}

Let us first discuss the simpler case of a Feynman amplitude in a scalar theory. The Feynman amplitude (6) has a complete Mellin representation [13], obtained in the following way. Let us rewrite the Symanzik polynomials as

$$
U(\alpha)=\sum_{j} \prod_{l=1}^{I} \alpha_{l}^{u_{l j}} \equiv \sum_{j} U_{j}, \quad N(\alpha)=\sum_{K} s_{K}\left(\prod_{l=1}^{I} \alpha_{l}^{n_{l K}}\right) \equiv \sum_{K} N_{K},
$$


where

$$
u_{l j}=\left\{\begin{array}{l}
0 \text { if the line } l \text { belongs to the } 1 \text {-tree } j \\
1 \text { otherwise }
\end{array}\right.
$$

and

$$
n_{l K}= \begin{cases}0 & \text { if the line } l \text { belongs to the } 2 \text {-tree } K \\ 1 & \text { otherwise. }\end{cases}
$$

Moreover,

$$
\sum_{l} u_{l j}=L ; \quad \sum_{l} n_{l K}=L+1
$$

for all $j$ and $K$, respectively.

The idea of the complete Mellin representation has its roots in the so-called 'multiple Mellin' representation introduced in [11]. In this case, the polynomial $N$ in eq. (6) is split into pieces, $N=\sum_{a} N_{a}$, in such a way that each piece $N_{a}$ has the property of being a FINE polynomial, that is, in each Hepp sector the orderings of the $\alpha$ 's induce one and only one dominant monomial of $N$ [12]. The point is that not all polynomials have the property of being FINE. Nevertheless, there always exists the solution of splitting the $N$ polynomial in all its monomials, which will always be FINE. In other words, if we adopt this extreme point of view, each piece $N_{a}$ is a monomial of $N$, which is necessarily FINE. In general, if the pieces $N_{a}$ are not the monomials of $N$, they will generate a 'multiple Mellin' representation of the amplitude [11]. In the extreme situation of splitting $N$ in all its monomials, $N=\sum_{K} N_{K}$, a 'complete Mellin' representation will be generated [13]. This is the point of view that we adopt here.

To proceed, let us remember the following theorem [17]: for a function $f(u)$, piecewise smooth for $u>0$, if the integral

$$
g(x)=\int_{0}^{\infty} d u u^{-x-1} f(u)
$$

is absolutely convergent for $\alpha<\operatorname{Re} x<\beta$, then

$$
f(u)=\frac{1}{2 \pi i} \int_{\sigma-i \infty}^{\sigma+i \infty} d x g(x) u^{x}
$$

with $\alpha<\sigma<\beta$.

Then, if we take $u=N_{K} / U$ in the above theorem, it is easy to see that

$$
e^{-N_{K} / U}=\int_{\tau_{K}} \Gamma\left(-y_{K}\right)\left(\frac{N_{K}}{U}\right)^{y_{K}},
$$

where $\int_{\tau_{K}}$ is a short notation for $\int_{-\infty}^{+\infty} \frac{d\left(\operatorname{Im} y_{K}\right)}{2 \pi i}$, with Re $y_{K}$ fixed at $\tau_{K}<0$. We may now recall the identity [18]

$$
\Gamma(u)(A+B)^{-u}=\int_{-\infty}^{\infty} \frac{d(\operatorname{Im} x)}{2 \pi i} \Gamma(-x) A^{x} \Gamma(x+u) B^{-x-u}
$$

and let us take $A \equiv U_{1}(x)$ and $B \equiv U_{2}+U_{3}+\cdots$. Using iteratively the identity above, it can be shown that, for $u=\sum_{K} y_{K}+d / 2$

$$
\Gamma\left(\sum_{K} y_{K}+\frac{d}{2}\right) U^{-\sum_{K} y_{K}-\frac{d}{2}}=\int_{\sigma} \prod_{j} \Gamma\left(-x_{j}\right) U_{j}^{x_{j}}
$$

with Re $x_{j}=\sigma_{j}<0, \operatorname{Re}\left(\sum_{K} y_{K}+\frac{d}{2}\right)=\sum_{K} \tau_{K}+\frac{d}{2}>0$, and $\int_{\sigma}$ means $\int_{-\infty}^{+\infty} \prod_{j} \frac{d\left(\operatorname{Im} x_{j}\right)}{2 \pi i}$ with $\sum_{j} x_{j}+\sum_{K} y_{K}=-\frac{d}{2}$. Then, after replacing eqs. (26) and (28) in eq. (6), the amplitude is written as

$$
I_{G}\left(s_{K}, m_{l}^{2}\right)=\int_{\Delta} \frac{\prod_{j} \Gamma\left(-x_{j}\right)}{\Gamma\left(-\sum_{j} x_{j}\right)} \prod_{K} s_{K}^{y_{K}} \Gamma\left(-y_{K}\right) \int_{0}^{\infty} \prod_{l} d \alpha_{l} \alpha_{l}^{\phi_{l}-1} e^{-\sum_{l} \alpha_{l} m_{l}^{2}},
$$


where

$$
\phi_{l} \equiv \sum_{j} u_{l j} \sigma_{j}+\sum_{K} n_{l K} \tau_{K}+1
$$

and $\Delta$ is the nonempty convex domain $\left(\sigma_{j}\right.$ and $\tau_{K}$ standing respectively for $\operatorname{Re}\left(-x_{j}\right)$ and $\left.\operatorname{Re}\left(-y_{K}\right)\right)$,

$$
\Delta=\left\{\begin{array}{l|l}
\sigma, \tau & \begin{array}{l}
\sigma_{j}<0 ; \tau_{K}<0 ; \sum_{j} x_{j}+\sum_{K} y_{K}=-\frac{d}{2} ; \\
\forall i, \operatorname{Re} \phi_{l} \equiv \sum_{j} u_{l j} \sigma_{j}+\sum_{K} n_{l K} \tau_{K}+1>0
\end{array}
\end{array}\right\}
$$

and the symbol $\int_{\Delta}$ means integration over the independent variables $\frac{\operatorname{Im} x_{j}}{2 \pi i}, \frac{\operatorname{Im} y_{K}}{2 \pi i}$.

The $\alpha$ integrations may be performed, using the well-known representation for the gamma function, so that we have

$$
\int_{0}^{\infty} d \alpha_{l} e^{-\alpha_{l} m_{l}^{2}} \alpha_{l}^{\phi_{l}-1}=\Gamma\left(\phi_{l}\right)\left(m_{l}^{2}\right)^{-\phi_{l}}
$$

and we finally get the complete Mellin representation of the amplitude in the scalar case:

$$
I_{G}\left(s_{K}, m_{l}^{2}\right)=\int_{\Delta} \frac{\prod_{j} \Gamma\left(-x_{j}\right)}{\Gamma\left(-\sum_{j} x_{j}\right)} \prod_{K} s_{K}^{y_{K}} \Gamma\left(-y_{K}\right) \prod_{l}\left(m_{l}^{2}\right)^{-\phi_{l}} \Gamma\left(\phi_{l}\right) .
$$

One should emphasize that the integral in eq. (29) above is assumed for simplicity to be ultraviolet convergent, but if this is not the case, it is shown in [13, 14] that the renormalization procedure does not alter the algebraic structure of integrands of the CM representation. It only changes the set of relevant integration domains in the Mellin variable space, all what follows remaining valid.

\section{THE COMPLETE MELLIN REPRESENTATION AND ASYMPTOTIC BEHAVIORS FOR GAUGE THEORIES}

Turning now to gauge theories, we see that in both equations for $I_{G}(\xi=1)$ and $I_{G}(\xi)$, given by eqs. (16) and (18), the integrals over the Schwinger parameters have the same form as in the scalar case in terms of the Symanzik polynomials, except for taking into account the substitutions $m_{i}^{2} \rightarrow \bar{m}_{i}^{2}$ and $s_{K} \rightarrow \bar{s}_{K}$ and having to perform the derivatives on the auxiliary variables $\zeta$ which modify $m_{i}^{2}$ and $s_{K}$ and are put to zero at the end of the calculation. Here $m_{l}$ stands generically for both the fermion masses and the infrared gauge-field regulator. The derivatives are always attached to Schwinger parameters, which then alter the power $\phi_{l}$ in the integrations corresponding to (32), a different alteration for each monomial of the $\mathcal{P}$ polynomial in the Feynman-gauge case, or of the $\mathcal{P}$ and $\mathcal{Q}_{1}$ and $\mathcal{Q}_{2}$ polynomials for $I_{G}(\xi)$.

For $I_{G}(\xi=1)$ in eq. (16), let us assume that the polynomial $\mathcal{P}$ has the form

$$
\mathcal{P}\left(-\frac{1}{\alpha_{i}} \frac{\partial}{\partial \zeta_{i}}\right)=\sum_{A} c_{A}\left(-\frac{1}{\alpha_{i}} \frac{\partial}{\partial \zeta_{i}}\right)^{d_{i}^{A}}
$$

Then, according to [14], for each monomial $\prod_{i}\left(-\frac{1}{\alpha_{i}} \frac{\partial}{\partial \zeta_{i}}\right)^{d_{i}^{A}}$ of the polynomial $\mathcal{P}$ the following complete Mellin representation holds:

$$
F_{A}^{(\xi=1)}\left(s_{K}, m_{l}^{2}\right)=\left.\int_{\Delta_{A}} \prod_{i}\left(-\frac{\partial}{\partial \zeta_{i}}\right)^{d_{i}^{A}} \frac{\prod_{j} \Gamma\left(-x_{j}\right)}{\Gamma\left(-\sum_{j} x_{j}\right)} \prod_{K} \bar{s}_{K}^{y_{K}} \Gamma\left(-y_{K}\right) \prod_{l}\left(\bar{m}_{l}^{2}\right)^{-\phi_{l}+d_{l}^{A}} \Gamma\left(\phi_{l}-d_{l}^{A}\right)\right|_{\zeta_{l}=0},
$$

where $\bar{m}_{l}$ stands for both the fermionic masses $\bar{m}_{i}$ and the infrared regulator $\mu$ of the gauge-field propagators; for the latter ones, $d_{l}^{A}=0$. Also, $\Delta_{A}$ is the modified nonempty convex domain

$$
\Delta_{A}=\left\{\begin{array}{l|l}
\sigma, \tau & \begin{array}{l}
\sigma_{j}<0 ; \tau_{K}<0 ; \sum_{j} x_{j}+\sum_{K} y_{K}=-\frac{d}{2} \\
\forall l, \operatorname{Re}\left(\phi_{l}-d_{l}^{A}\right) \equiv \sum_{j} u_{l j} \sigma_{j}+\sum_{K} n_{l K} \tau_{K}-d_{l}^{A}+1>0
\end{array}
\end{array}\right\}
$$

We see that now the derivatives may be taken out of the integration, so that we define

$$
F_{A}^{(\xi=1)}\left(s_{K}, m_{l}^{2}\right)=\left.\prod_{l}\left(-\frac{\partial}{\partial \zeta_{l}}\right)^{d_{l}^{A}} F_{A}^{(\xi=1)}\left(\bar{s}_{K}, \bar{m}_{l}^{2}\right)\right|_{\zeta_{l}=0}
$$


with

$$
F_{A}^{(\xi=1)}\left(\bar{s}_{K}, \bar{m}_{l}^{2}\right)=\int_{\Delta_{A}} \frac{\prod_{j} \Gamma\left(-x_{j}\right)}{\Gamma\left(-\sum_{j} x_{j}\right)} \prod_{K} \bar{s}_{K}^{y_{K}} \Gamma\left(-y_{K}\right) \prod_{l}\left(\bar{m}_{l}^{2}\right)^{-\phi_{l}+d_{l}^{A}} \Gamma\left(\phi_{l}-d_{l}^{A}\right) .
$$

In this case, a general asymptotic regime is defined by scaling the invariants $\bar{s}_{K}$ and $\bar{m}_{l}^{2}$,

$$
\begin{aligned}
& \bar{s}_{K} \rightarrow \lambda^{a_{K}} \bar{s}_{K}, \\
& \bar{m}_{l}^{2} \rightarrow \lambda^{a_{l}} \bar{m}_{l}^{2},
\end{aligned}
$$

where $a_{K}$ and $a_{l}$ may have positive, negative, or null values, and letting $\lambda$ go to infinity. We then obtain under this scaling

$$
F_{A}^{(\xi=1)}\left(\lambda, \bar{s}_{K}, \bar{m}_{l}^{2}\right)=\left.\int_{\Delta_{A}} \frac{\prod_{j} \Gamma\left(-x_{j}\right)}{\Gamma\left(-\sum_{j} x_{j}\right)} \prod_{K} \bar{s}_{K}^{y_{K}} \Gamma\left(-y_{K}\right) \prod_{l}\left(\bar{m}_{l}^{2}\right)^{-\phi_{l}+d_{l}^{A}} \Gamma\left(\phi_{l}-d_{l}^{A}\right) \lambda^{\psi^{A}}\right|_{\zeta_{l}=0},
$$

where the exponent of $\lambda$ is the following linear function of the Mellin variables:

$$
\psi^{A}=\sum_{K} a_{K} y_{K}-\sum_{l} a_{l}\left[\phi_{l}\left(x_{j}, y_{K}\right)-d_{l}^{A}\right] .
$$

Then the proof of the theorem given in [11] for functions of the form (33) can be extended for (38), and the following theorem is valid: as $\lambda \rightarrow \infty$, we have an asymptotic expansion of the integral $F_{A}^{(\xi=1)}\left(\lambda, \bar{s}_{K}, \bar{m}_{l}^{2}\right)$ of the form

$$
F_{A}^{(\xi=1)}\left(\lambda, \bar{s}_{K}, \bar{m}_{l}^{2}\right)=\sum_{p=p_{\max }}^{-\infty} \sum_{q=0}^{q_{\max }(p)} F_{p q}^{(\xi=1) A}\left(\bar{s}_{K}, \bar{m}_{l}^{2}\right) \lambda^{p} \ln ^{q} \lambda
$$

where $p$ runs over the rational values of a decreasing arithmetic progression, with $p_{\max }$ as a 'leading power', and $q$, for a given $p$, runs over a finite number of nonnegative integer values.

A systematic way of evaluating the coefficients $F_{p q}$ can be done by successive analytical continuations of the linear form $\psi$ to "smaller" cells of the type $\Delta$ in eq. (31). Under the scaling (39), and summing over all monomials, $I_{G}(\xi=1)$ in eq. (16) becomes

$$
\begin{aligned}
I_{G}(\xi=1, \lambda) & =\left.C_{G} \sum_{A} c_{A} \prod_{l}\left(-\frac{\partial}{\partial \zeta_{l}}\right)^{d_{l}^{A}} \sum_{p=p_{\max }}^{-\infty} \sum_{q=0}^{q_{\max }(p)}\left[F_{p q}^{(\xi=1) A}\left(\bar{s}_{K}, \bar{m}_{l}^{2}\right)\right] \lambda^{p} \ln ^{q} \lambda\right|_{\zeta_{l}=0} \\
& =\left.C_{G} \sum_{p=p_{\max }} \sum_{q=0}^{q_{\max }^{(p)}}\left[\sum_{A} c_{A} \prod_{l}\left(-\frac{\partial}{\partial \zeta_{l}}\right)^{d_{l}^{A}} F_{p q}^{(\xi=1) A}\left(\bar{s}_{K}, \bar{m}_{l}^{2}\right)\right] \lambda^{p} \ln ^{q} \lambda\right|_{\zeta_{l}=0} \\
& =C_{G} \sum_{p=p_{\max }} \sum_{q=0}^{q_{\max }(p)} G_{p q}^{(\xi=1)}\left(s_{K} ; m_{l}^{2}\right) \lambda^{p} \ln ^{q} \lambda
\end{aligned}
$$

where we have defined

$$
G_{p q}^{(\xi=1)}\left(s_{K} ; m_{l}^{2}\right)=\left.\sum_{A} c_{A} \prod_{l}\left(-\frac{\partial}{\partial \zeta_{l}}\right)^{d_{l}^{A}} F_{p q}^{(\xi=1) A}\left(\bar{s}_{K}, \bar{m}_{l}^{2}\right)\right|_{\zeta_{l}=0}
$$

For $I_{G}(\xi)$, generalizing what has been done for eq. (35), we consider the product of polynomials $\mathcal{P}, \mathcal{Q}_{1}$ and $\mathcal{Q}_{2}$ in eq. (18) in the form

$$
\mathcal{P} \mathcal{Q}_{1} \mathcal{Q}_{2}=\sum_{B} c_{B}(\xi) \prod_{i}\left(-\frac{1}{\alpha_{i}} \frac{\partial}{\partial \zeta_{i}}\right)^{d_{i}^{B}}\left(-\frac{1}{\beta_{r_{1}}} \frac{\partial}{\partial \zeta_{r_{1}}}\right)^{e_{r_{1}}^{B}}\left(-\frac{1}{\beta_{r_{2}}} \frac{\partial}{\partial \zeta_{r 2}}\right)^{e_{r_{2}}^{B}}
$$

In order to proceed, notice that eq. (20) is modified in the present case to

$$
\begin{aligned}
U(\alpha, \beta) & =\sum_{j} \prod_{i \in F} \alpha_{i}^{u_{i j}} \prod_{r_{1} \in \Gamma} \beta_{r_{1}}^{v_{r_{1} j}} \prod_{r_{2} \in \Gamma} \beta_{r_{2}}^{v_{r_{2} j}} \\
N(\alpha, \beta) & =\sum_{K} \bar{s}_{K} \prod_{i \in F} \alpha_{i}^{n_{i j}} \prod_{r_{1} \in \Gamma} \beta_{r_{1}}^{m_{r_{1} K}} \prod_{r_{2} \in \Gamma} \beta_{r_{2}}^{m_{r_{2} K}} .
\end{aligned}
$$


We now replace the above expressions in eq. (18), together with eqs. (26) and (28). After some manipulations, we find

$$
\begin{aligned}
I_{G}(\xi)= & C_{G} \sum_{B} c_{B}(\xi) \int_{\Delta_{B}} \frac{\prod_{j} \Gamma\left(-x_{j}\right)}{\Gamma\left(-\sum_{j} x_{j}\right)} \prod_{K} \Gamma\left(-y_{K}\right) \\
& \times\left\{\prod_{i}\left(-\frac{\partial}{\partial \zeta_{i}}\right)^{d_{i}^{B}} \int d \alpha_{i} e^{-\sum_{i} \alpha_{i} \bar{m}_{i}^{2}} \alpha_{i}^{\phi_{i}-d_{i}^{B}-1}\right. \\
& \times \prod_{r_{1}}\left(-\frac{\partial}{\partial \zeta_{r_{1}}}\right)^{e_{r_{1}}^{B}} \int d \beta_{r_{1}} e^{-\sum_{r_{1}} \beta_{r_{1}} \bar{\mu}_{r_{1}}^{2} \alpha_{i}^{\phi_{r_{1}}-e_{r_{1}}^{B}-1}} \\
& \times \prod_{r_{2}}\left(-\frac{\partial}{\partial \zeta_{r_{2}}}\right)^{e_{r_{2}}^{B}} \int d \beta_{r_{2}} e^{\left.-\sum_{r_{2}} \beta_{r_{2}} \bar{\mu}_{r_{2}}^{2} \alpha_{i}^{\phi_{r_{2}}-e_{r_{2}}^{B}-1}\right\}\left.\bar{s}_{K}^{y_{K}}\right|_{\zeta=0}},
\end{aligned}
$$

where $\phi_{i}=\sum_{j} u_{i j} x_{j}+\sum_{K} n_{i K} y_{K}$ as before, and

$$
\phi_{r_{1,2}}=\sum_{j} v_{j r_{1,2}} x_{j}+\sum_{K} m_{K r_{1,2}} y_{K}
$$

and now the domain of integration is

$$
\Delta_{B}=\left\{\begin{array}{l|l}
\sigma, \tau & \begin{array}{l}
\sigma_{j}<0 ; \tau_{K}<0 ; \sum_{j} x_{j}+\sum_{K} y_{K}=-\frac{d}{2} \\
\forall i, \operatorname{Re}\left(\phi_{i}-d_{i}^{B}\right) \equiv \sum_{j} u_{i j} \sigma_{j}+\sum_{K} n_{i K} \tau_{K}-d_{i}^{B}+1>0 \\
\forall r_{1}, r_{2}, \operatorname{Re}\left(\phi_{r_{1,2}}-e_{r_{1,2}}^{B}\right) \equiv \sum_{j} v_{j r_{1,2}} \sigma_{j}+\sum_{K} m_{K r_{1,2}} \tau_{K}-e_{r_{1,2}}^{B}+1>0
\end{array}
\end{array}\right\}
$$

The integrals over all Schwinger parameters $\alpha$ and $\beta$ are performed in the same fashion as in eq. (32), so the amplitude is written as

$$
\begin{aligned}
I_{G}(\xi)= & C_{G} \sum_{B} c_{B}(\xi) \prod_{i}\left(-\frac{\partial}{\partial \zeta_{i}}\right)^{d_{i}^{B}} \prod_{r_{1}}\left(-\frac{\partial}{\partial \zeta_{r_{1}}}\right)^{e_{r_{1}}^{B}} \prod_{r_{2}}\left(-\frac{\partial}{\partial \zeta_{r_{2}}}\right)^{e_{r_{2}}^{B}} \\
& \times \int_{\Delta_{B}} \frac{\prod_{j} \Gamma\left(-x_{j}\right)}{\Gamma\left(-\sum_{j} x_{j}\right)} \prod_{K} \bar{s}_{K}^{y_{K}} \Gamma\left(-y_{K}\right) \\
& \times \Gamma\left(\phi_{i}-d_{i}^{B}\right)\left(\bar{m}_{i}^{2}\right)^{-\phi_{i}+d_{i}^{B}} \Gamma\left(\phi_{r_{1}}-e_{r_{1}}^{B}\right)\left(\bar{\mu}_{r_{1}}^{2}\right)^{-\phi_{r_{1}}+e_{r_{1}}^{B}} \Gamma\left(\phi_{r_{2}}-e_{r_{2}}^{B}\right)\left(\bar{\mu}_{r_{2}}^{2}\right)^{-\phi_{r_{2}}+\left.e_{r_{2}}^{B}\right|_{\zeta=0}}
\end{aligned}
$$

where $j$ runs over the whole set of monomials of $U(\alpha, \beta)$, eq. (46). Then, performing steps similar to those that led to eq. (37) for $I_{G}(\xi=1)$, we get for each monomial of the product of polynomials $\mathcal{P} \mathcal{Q}_{1} \mathcal{Q}_{2}$ in eq. (45) the expression

$$
F_{B}\left(s_{K}, m_{i}^{2}, \mu^{2}\right)=\left.\prod_{i}\left(-\frac{\partial}{\partial \zeta_{i}}\right)^{d_{i}^{B}} \prod_{r_{1}}\left(-\frac{\partial}{\partial \zeta_{r_{1}}}\right)^{e_{r_{1}}^{B}} \prod_{i}\left(-\frac{\partial}{\partial \zeta_{r_{2}}}\right)^{e_{r_{2}}^{B}} F_{B}\left(\bar{s}_{K}, \bar{m}_{i}^{2}, \bar{\mu}_{r_{1,2}}^{2}\right)\right|_{\zeta=0}
$$

where

$$
F_{B}\left(\bar{s}_{K}, \bar{m}_{i}^{2}, \bar{\mu}_{r_{1,2}}^{2}\right)=\int_{\Delta_{B}} \frac{\prod_{j} \Gamma\left(-x_{j}\right)}{\Gamma\left(-\sum_{j} x_{j}\right)} \prod_{K} \bar{s}_{K}^{y_{K}} \Gamma\left(-y_{K}\right) \prod_{l}\left(\bar{m}_{l}^{2}\right)^{-\phi_{l}+d_{l}^{B}} \Gamma\left(\phi_{l}-d_{l}^{B}\right) .
$$

We now consider an asymptotic regime defined by

$$
\begin{aligned}
\bar{s}_{K} & \rightarrow \lambda^{a_{K}} \bar{s}_{K}, \\
\bar{m}_{i}^{2} & \rightarrow \lambda^{a_{i}} \bar{m}_{i}^{2}, \\
\bar{\mu}_{r_{1,2}}^{2} & \rightarrow \lambda^{a_{r_{1,2}}} \bar{\mu}_{r_{1,2}}^{2}
\end{aligned}
$$

where $a_{K}, a_{i}$ and $a_{r_{1,2}}$ may have positive, negative, or null values, and we let $\lambda$ go to infinity. Under the above scaling, $F_{B}$ in eq. (53) then becomes a function of $\lambda$, given by

$$
F_{B}\left(\bar{s}_{K}, \bar{m}_{i}^{2}, \bar{\mu}_{r_{1,2}}^{2}\right)=\int_{\Delta_{B}} \frac{\prod_{j} \Gamma\left(-x_{j}\right)}{\Gamma\left(-\sum_{j} x_{j}\right)} \prod_{K} \bar{s}_{K}^{y_{K}} \Gamma\left(-y_{K}\right) \prod_{l}\left(\bar{m}_{l}^{2}\right)^{-\phi_{l}+d_{l}^{B}} \Gamma\left(\phi_{l}-d_{l}^{B}\right) \lambda^{\psi^{B}},
$$


with the exponent of $\lambda$ being

$$
\psi^{B}=\sum_{K} a_{K} y_{K}-\sum_{i} a_{i}\left(x_{j}, y_{K}\right)-d_{i}^{B}-\sum_{r_{1}} a_{r_{1}}\left(x_{j}, y_{K}\right)-e_{r_{1}}^{B}-\sum_{r_{2}} a_{r_{2}}\left(x_{j}, y_{K}\right)-e_{r_{2}}^{B} .
$$

Since the integral in the function (55) has the same form of that of the corresponding one in the scalar field theory, the following asymptotic behavior expansion holds:

$$
F_{B}\left(\lambda, \bar{s}_{K}, \bar{m}_{i}^{2}, \bar{\mu}_{r_{1,2}}^{2}\right)=\sum_{p=p_{\max }}^{-\infty} \sum_{q=0}^{q_{\max }(p)} F_{p q}^{B}\left(\bar{s}_{K}, \bar{m}_{i}^{2}, \bar{\mu}_{r_{1,2}}^{2}\right) \lambda^{p} \ln ^{q} \lambda
$$

Therefore, from (55),

$$
I_{G}(\lambda, \xi)=C_{G} \sum_{p=p_{\max }}^{-\infty} \sum_{q=0}^{q_{\max }(p)} G_{p q}\left(\xi ; s_{K}^{(2)} ; m_{i}^{2}, \mu^{2}\right) \lambda^{p} \ln ^{q} \lambda
$$

where

$$
G_{p q}\left(\xi ; s_{K} ; m_{i}^{2}, \mu^{2}\right)=\left.\sum_{B} c_{B}(\xi) \prod_{i}\left(-\frac{\partial}{\partial \zeta_{i}}\right)^{d_{i}^{B}} \prod_{r_{1}}\left(-\frac{\partial}{\partial \zeta_{r_{1}}}\right)^{e_{r_{1}}^{B}} \prod_{i}\left(-\frac{\partial}{\partial \zeta_{r_{2}}}\right)^{e_{r_{2}}^{B}} F_{p q}^{B}\left(\bar{s}_{K}, \bar{m}_{i}^{2}, \bar{\mu}_{r_{1,2}}^{2}\right)\right|_{\zeta=0} .
$$

\section{CONCLUDING REMARKS}

We have stated a theorem which generalizes for gauge field theories in an unspecified gauge previous results on the asymptotic behaviors of scalar field theories. Its relevance stems from physical situations in which the choice of gauges other than the Feynman gauge is mandatory. These situations do exist, as we have mentioned in the introduction. The proof of the theorem has been possible by conveniently changing the invariants $s_{K}, m_{i}^{2}$ and $\mu^{2}$ into new objects $\bar{s}_{K}, \bar{m}_{i}^{2}$ and $\bar{\mu}_{r_{1,2}}^{2}$ by means of dummy variables $\zeta$ associated to each internal line of the diagram, to be taken equal to zero in the end. It results that the relevant Feynman integrals are expressed as polynomials of derivatives with respect to this dummy variables, acting on integrals over the Schwinger parameters. Complete Mellin representations can be written for gauge theory amplitudes and a previous theorem for asymptotic behaviors of scalar amplitudes was generalized for them. It results that the dependence on the gauge-fixing parameter is contained in the coefficients of the asymptotic expansion.

We recall that the idea of the complete Mellin representation is an extreme version of the multiple Mellin' representation [11]. In this case, the polynomial $N$ is split into FINE pieces, that is, in each Hepp sector the orderings of the Schwinger variables induce one and only one dominant monomial of $N$. The fact is that not all polynomials have the property of being FINE, and in some cases, the only solution is to split the $N$ polynomial in all its monomials, which will always be FINE, and generates the complete Mellin representation.

A point to be emphasized is the following. One may wonder why such an involved method should be used in order to obtain an asymptotic expansion of Feynman amplitudes, at the price of employing a rather cumbersome notation, instead of using the much simpler technique of the Mellin transform. The answer is that the Mellin transform method, which is equivalent to a simple Mellin representation, is valid only if the scaled part of the Symanzik polynomial $N$ has the property of being FINE. This is the case for simple (low-order) Feynman diagrams, but it is not true in general for an arbitrary diagram. For all those high-order diagrams, whose inner complexity leads to a non-FINE $N$ polynomial, and for which the Mellin transform (or, equivalently, the simple Mellin representation) method does not apply, our theorem guarantees the existence of the asymptotic expansions with the same structure as that of the simpler FINE cases.

\section{Acknowledgments}

This work received partial financial support from $\mathrm{CNPq} / \mathrm{MCT}$ (Brazil). I.R. acknowledges partial financial support from Pronex/FAPERJ.

[1] S. Friot, D. Greynat and E. de Rafael, Phys. Lett. B 628 (2005) 73. 
[2] V.N. Gribov, Nucl. Phys. B 139 (1978) 1; D. Zwanziger, Nucl. Phys. B 399 (1993) 477.

[3] D. Dudal, R.F. Sobreiro, S.P. Sorella and H. Verschelde, Phys. Rev. D 72 (2005) 014016; J.A. Gracey, J. High Energy Physics 05 (2006) 052.

[4] A.F. Ferrari, H.O. Girotti, M. Gomes, A.Yu. Petrov, A. Ribeiro, V.O. Rivelles and A.J. da Silva, Phys. Rev. D 69 (2004) 025008; hep-th/0309154

[5] M.C. Bergère and J.B. Zuber, Commun. Math. Phys. 35 (1974) 113.

[6] M.C. Bergère and Y.-M.P. Lam, Commun. Math. Phys. 39 (1974) 1.

[7] M.C. Bergère and Y.-M.P. Lam, J. Math. Phys. 17 (1976) 1546.

[8] N. Nakanishi, Graph Theory and Feynman Integrals, Gordon and Breach, New York, 1971.

[9] C. Itzykson and J.B. Zuber, Quantum Field Theory, McGraw-Hill, New York, 1980.

[10] M.C. Bergère and Y.-M.P. Lam, preprint, Freie Universität, Berlin, HEP May 1979/9 (unpublished).

[11] M.C. Bergère, C. de Calan and A.P.C. Malbouisson, Commun. Math. Phys. 62 (1978) 137.

[12] K. Hepp, Commun. Math. Phys. 2 (1966) 301.

[13] C. de Calan and A.P.C. Malbouisson, Ann. Inst. Henri Poincaré 32 (1980) 91.

[14] C. de Calan, F. David and V. Rivasseau, Commun. Math. Phys. 78 (1981) 531.

[15] C. de Calan and A.P.C. Malbouisson, Commun. Math. Phys. 90 (1983) 413.

[16] A.P.C. Malbouisson, J. Phys. A: Math. Gen. 33 (2000) 3587; "Critical behavior of correlation functions and asymptotic expansions of Feynman amplitudes", in Fluctuating Paths and Fields: Festschrift Dedicated to Hagen Kleinert, W. Janke, A. Pelster, H.-J. Schmidt and M. Bachmann (eds.), World Scientific, Singapore, 2001.

[17] R. Courant and D. Hilbert, Methods of Mathematical Physics, Vol. 1, Springer, Berlin, 1968, p. 104.

[18] I.S. Gradzhteyn and I.M. Ryzhik, Table of Integrals, Series and Products, Academic Press, New York, 1965, p. 657. 